(C) 2003 American Chemical Society, Organometallics, Zurawinski om030343g Supporting Info Page 1

\title{
Chiral Phosphino-(sulfinylmethyl)triarylphosphonium Ylide Ligands: Rhodium Complexes and Catalytic Properties
}

Remigiusz Zurawinski, Bruno Donnadieu, Marian Mikolajczyk,* Remi Chauvin*

\section{SUPPORTING INFORMATION}

- X-ray crystal structure data of complex $\left[5 \mathbf{5 a}^{-}\left[\mathrm{PF}_{6}\right]\right.$

$-{ }^{1} \mathrm{H},{ }^{31} \mathrm{P}$ and ${ }^{13} \mathrm{C}$ NMR spectra of compounds $[4 \mathrm{bH}]\left[\mathrm{PF}_{6}\right],[4 \mathrm{cH}]\left[\mathrm{PF}_{6}\right],[5 \mathrm{a}]\left[\mathrm{PF}_{6}\right]$ and $[5 \mathrm{~b}]\left[\mathrm{PF}_{6}\right]$

Table S1 .Crystal data, data collection and structure refinement

\begin{tabular}{ll}
\hline Empirical formula & $\mathrm{C}_{46} \mathrm{H}_{44} \mathrm{~F}_{6} \mathrm{OP}_{3} \mathrm{SRh}$ \\
Formula weight & 954.69 \\
Temperature & $180(2) \mathrm{K}$ \\
Wavelength & $0.71073 \AA$ \\
Crystal system, space group & orthorhombic, P 212121 \\
Unit cell dimensions & $\mathrm{a}=14.561(5) \AA$ \\
& $\mathrm{b}=14.596(5) \AA$ \\
Volume & $8421(4) \AA 3$ \\
Z, Calculated density & $8,1.506 \mathrm{mg} / \mathrm{m} 3$ \\
Absorption coefficient & $0.632 \mathrm{~mm}-1$ \\
F(000) & 3904 \\
Crystal size & $(0.42 \times 0.35 \times 0.14) \mathrm{mm}$ \\
Crystal form & parallelepiped \\
Crystal color & $0 \mathrm{range}$ \\
Tube power & $1.50 \mathrm{~kW}$ \\
Tube voltage & $50 \mathrm{kV}$ \\
Tube current & $30 \mathrm{~mA}$ \\
Collimator size & $0.5 \mathrm{~mm}$ \\
Detector distance & $70.0 \mathrm{~mm}$ \\
2theta range & $(3.3-52.1)^{\circ}$ \\
& 1
\end{tabular}


(C) 2003 American Chemical Society, Organometallics, Zurawinski om030343g Supporting Info Page 2

d (hkl) range

Phi movement mode

range for data collection

Index ranges

Reflections collected / unique

Completeness to 2 theta $=56.56$

Refinement method

Data / restraints / parameters

Absorption corrections

Tmin - Tmax

Goodness-of-fit on F2

Scheme of ponderation

where $\mathrm{P}=(\mathrm{Fo} 2+2 \mathrm{FC} 2) / 3$

Final $R$ indices $[I>2(I)]$

$\mathrm{R}$ indices (all data)

Absolute structure parameter

Largest diff. peak and hole
$(12.453-0.809) \AA$

rotation

$$
\begin{aligned}
& (3.47 \text { to } 28.28)^{\circ} \\
& -19<=\mathrm{h}<=19,-19<=\mathrm{k}<=16,-52<=1<=52 \\
& 68906 / 10449[\mathrm{R}(\mathrm{int})=0.0693] \\
& 99.5 \% \\
& \text { Full-matrix least-squares on } \mathrm{F} 2 \\
& 10449 / 0 / 528 \\
& \text { semi-empirical } \\
& 0.615-0.886 \\
& 1.091 \\
& \text { weight }=1 /[2(\mathrm{Fo} 2)+(0.0188 \mathrm{P}) 2+16.526 \mathrm{P}] \\
& \text { R1 }=0.0451, \text { wR2 }=0.0877 \\
& \text { R1 }=0.0486, \text { wR2 }=0.0897 \\
& 0.01(3) \\
& (0.715 \text { and }-0.495) \mathrm{A}-3
\end{aligned}
$$

${ }^{*}$ DIFABS - N. Walker and D. Stuart, Acta Crystallogr., Sect A 1983, 39, 158-166.

Table S2. Atomic coordinates (x104) and equivalent isotropic displacement parameters $(\AA 2 x 103), U(e q)$ is defined as one third of the trace of the orthogonalized Uij tensor

\begin{tabular}{lllll} 
& $\mathbf{x}$ & $\mathbf{y}$ & $\mathbf{U}(\mathbf{e q})$ \\
\hline $\mathrm{Rh}$ & $7876(1)$ & $8535(1)$ & $2038(1)$ & $20(1)$ \\
$\mathrm{S}(1)$ & $6039(1)$ & $8797(1)$ & $2506(1)$ & $28(1)$ \\
$\mathrm{P}(1)$ & $6650(1)$ & $8284(1)$ & $1695(1)$ & $20(1)$ \\
$\mathrm{P}(2)$ & $6919(1)$ & $6993(1)$ & $2408(1)$ & $23(1)$ \\
$\mathrm{O}(1)$ & $5414(2)$ & $8393(2)$ & $2765(1)$ & $42(1)$ \\
$\mathrm{C}(1)$ & $7107(2)$ & $8170(2)$ & $2482(1)$ & $21(1)$ \\
$\mathrm{C}(2)$ & $6481(3)$ & $9816(3)$ & $2695(1)$ & $30(1)$ \\
$\mathrm{C}(3)$ & $6427(3)$ & $10629(3)$ & $2519(1)$ & $41(1)$ \\
$\mathrm{C}(4)$ & $6718(4)$ & $11428(3)$ & $2677(1)$ & $52(1)$ \\
$\mathrm{C}(5)$ & $7049(3)$ & $11418(3)$ & $3003(1)$ & $52(1)$ \\
$\mathrm{C}(6)$ & $7090(4)$ & $10596(4)$ & $3174(1)$ & $48(1)$ \\
$\mathrm{C}(7)$ & $6807(3)$ & $9791(3)$ & $3023(1)$ & $37(1)$
\end{tabular}


(C) 2003 American Chemical Society, Organometallics, Zurawinski om030343g Supporting Info Page 3

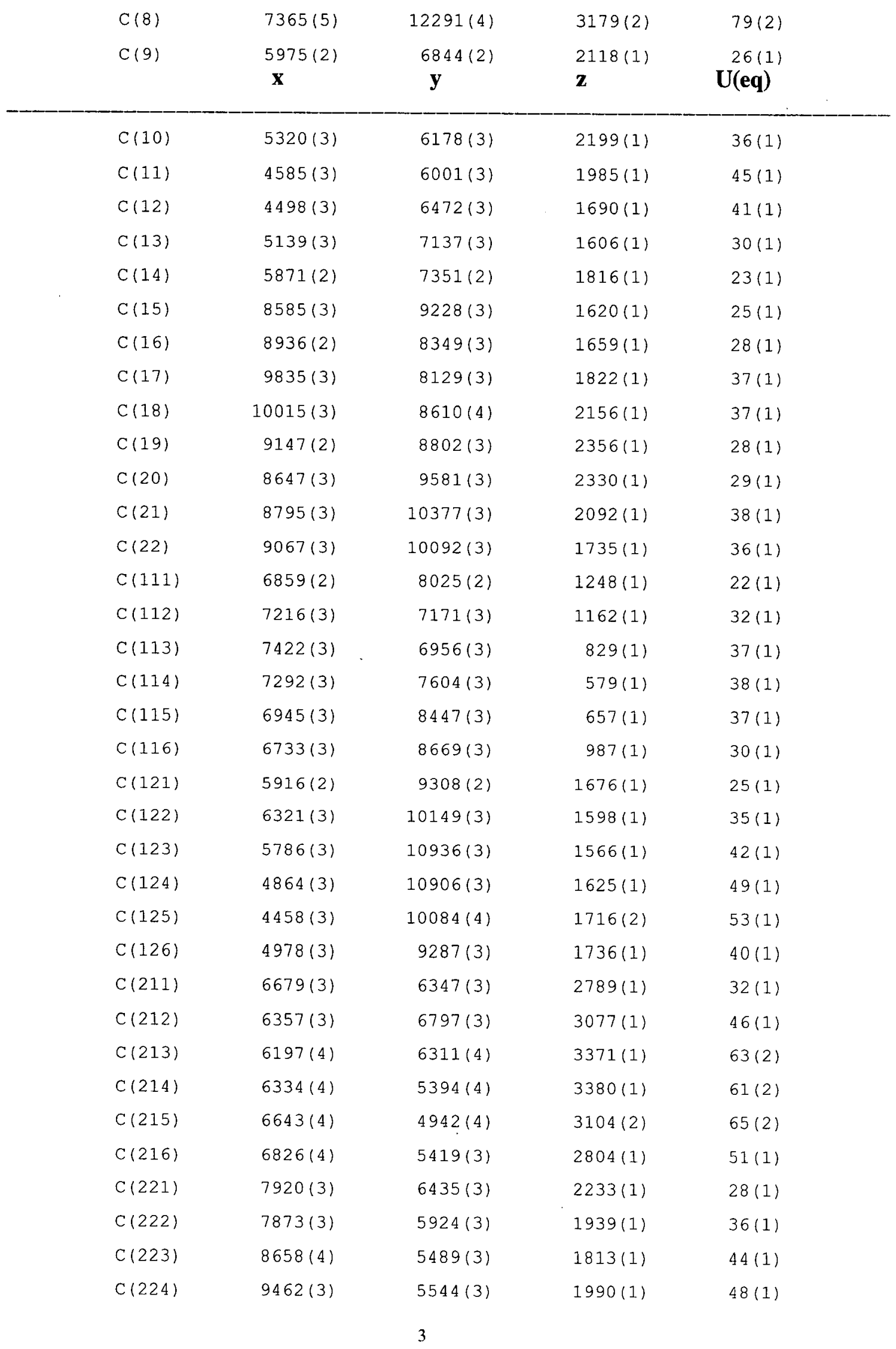


(C) 2003 American Chemical Society, Organometallics, Zurawinski om030343g Supporting Info Page 4

$\begin{array}{lllll}\mathrm{C}(225) & 9506(3) & 6020(3) & 2294(1) & 45(1) \\ \mathrm{C}(226) & 8735(3) & 6460(3) & 2417(1) & 37(1)\end{array}$

$\star \mathrm{PF} 6$

$\begin{array}{llll}\mathbf{x} & \mathbf{y} & \mathbf{U}(\mathbf{e q})\end{array}$

$\begin{array}{lrrrr}P(3) & 6844(1) & 3961(1) & 927(1) & 43(1) \\ F(1) & 6509(3) & 3148(3) & 1156(1) & 98(2) \\ F(2) & 7403(3) & 3298(3) & 696(1) & 86(1) \\ F(3) & 7705(3) & 4114(4) & 1154(1) & 112(2) \\ F(4) & 7168(3) & 4764(2) & 678(1) & 88(1) \\ F(5) & 6250(3) & 4682(4) & 1124(1) & 127(2) \\ F(6) & 5977(2) & 3792(2) & 692(1) & 71(1)\end{array}$

Table S3. Full list of bond lengths $[\AA]]$ and angles $\left[{ }^{\circ}\right]$, e.s.d's in parentheses refers to the last significant digit

$\begin{array}{llllll}R h & -C(1) & 2.152(3) & R h & -C(16) & 2.173(3) \\ R h & -C(15) & 2.199(3) & R & -C(20) & 2.221(4) \\ R h & -C(19) & 2.272(3) & R h & -P(1) & 2.2730(10) \\ S(1) & -O(1) & 1.495(3) & S(1) & -C(2) & 1.786(4) \\ S(1) & -C(1) & 1.806(4) & P(1) & -C(14) & 1.835(3) \\ P(1) & -C(121) & 1.839(4) & P(1) & -C(111) & 1.839(4) \\ P(2) & -C(1) & 1.764(4) & P(2) & -C(221) & 1.808(4) \\ P(2) & -C(9) & 1.808(4) & P(2) & -C(211) & 1.814(4) \\ C(1) & -H(1) & 0.85(4) & C(2) & -C(3) & 1.378(6) \\ C(2) & -C(7) & 1.386(6) & C(3) & -C(4) & 1.390(7) \\ C(4) & -C(5) & 1.380(8) & C(5) & -C(6) & 1.379(7) \\ C(5) & -C(8) & 1.523(7) & C(6) & -C(7) & 1.381(6) \\ C(9) & -C(10) & 1.399(5) & C(9) & -C(14) & 1.414(5) \\ C(10) & -C(11) & 1.389(6) & C(11) & -C(12) & 1.364(6) \\ C(12) & -C(13) & 1.386(5) & C(13) & -C(14) & 1.389(5) \\ C(15) & -C(16) & 1.389(6) & C(15) & -C(22) & 1.514(5) \\ & & & & & \end{array}$




$\begin{array}{llll}C(16)-C(17) & 1.495(5) & C(17)-C(18) & 1.518(6) \\ C(18)-C(19) & 1.518(5) & C(19)-C(20) & 1.355(6) \\ C(20)-C(21) & 1.511(5) & C(21)-C(22) & 1.526(6) \\ C(111)-C(112) & 1.393(5) & C(111)-C(116) & 1.407(5) \\ C(112)-C(113) & 1.388(5) & C(113)-C(114) & 1.384(6) \\ C(114)-C(115) & 1.366(6) & C(115)-C(116) & 1.383(5) \\ C(121)-C(126) & 1.386(5) & C(121)-C(122) & 1.398(5) \\ C(122)-C(123) & 1.394(6) & C(123)-C(124) & 1.363(7) \\ C(124)-C(125) & 1.386(7) & C(125)-C(126) & 1.390(6) \\ C(211)-C(216) & 1.372(6) & C(211)-C(212) & 1.397(6) \\ C(212)-C(213) & 1.383(6) & C(213)-C(214) & 1.354(8) \\ C(214)-C(215) & 1.357(9) & C(215)-C(216) & 1.403(7) \\ C(221)-C(222) & 1.386(5) & C(221)-C(226) & 1.394(6) \\ C(222)-C(223) & 1.399(6) & C(223)-C(224) & 1.366(7) \\ C(224)-C(225) & 1.393(7) & C(225)-C(226) & 1.382(6)\end{array}$

\section{$\star P F 6$}

$\begin{array}{llll}P(3)-F(3) & 1.559(4) & P(3)-F(2) & 1.561(3) \\ P(3)-F(5) & 1.571(4) & P(3)-F(1) & 1.571(3) \\ P(3)-F(6) & 1.588(3) & P(3)-F(4) & 1.602(4)\end{array}$

\begin{tabular}{|c|c|c|c|c|c|c|c|}
\hline$C(1)$ & $-R h$ & $-C(16)$ & $154.79(14)$ & $C(1)$ & - $R h$ & $-c(15)$ & $166.94(13)$ \\
\hline$C(16)$ & $-R h$ & $-C(15)$ & $37.06(15)$ & $C(1)$ & $-R h$ & $-C(20)$ & $90.45(13)$ \\
\hline$C(16)$ & - Rh & $-C(20)$ & $95.01(14)$ & $C(15)$ & $-\mathrm{Rh}$ & $-C(20)$ & $80.72(14)$ \\
\hline$C(1)$ & - Rh & $-C(19)$ & $90.78(14)$ & $C(16)$ & - $R h$ & $-C(19)$ & $79.99(14)$ \\
\hline$C(15)$ & - Rh & $-C(19)$ & $87.49(14)$ & $C(20)$ & $-R h$ & $-C(19)$ & $35.08(14)$ \\
\hline$C(1)$ & $-\mathrm{Rh}$ & $-P(1)$ & $92.26(10)$ & $C(16)$ & $-\mathrm{Rh}$ & $-P(1)$ & $97.13(10)$ \\
\hline$C(15)$ & - Rh & $-P(1)$ & $89.59(10)$ & $C(20)$ & $-R h$ & $-P(1)$ & $145.02(11)$ \\
\hline$C(19)$ & - $R h$ & $-P(1)$ & $176.94(10)$ & $O(1)$ & $-S(1)$ & $-C(2)$ & $104.99(18)$ \\
\hline$O(1)$ & $-S(1)$ & $-C(1)$ & $111.07(17)$ & $C(2)$ & $-S(1)$ & $-C(1)$ & $97.65(17)$ \\
\hline$C(14)$ & $-P(1)$ & $-C(121)$ & $104.74(16)$ & $C(14)$ & $-P(1)$ & $-C(111)$ & $101.58(16)$ \\
\hline$C(121)$ & $-P(1)$ & $-C(111)$ & $102.91(16)$ & $C(14)$ & $-P(1)$ & $-\mathrm{Rh}$ & $116.70(12)$ \\
\hline$C(121)$ & $-P(1)$ & $-R h$ & $110.49(12)$ & $C(111)$ & $-P(1)$ & - $R h$ & $118.68(12)$ \\
\hline$C(1)$ & $-P(2)$ & $-C(221)$ & $112.20(17)$ & $C(1)$ & $-P(2)$ & $-C(9)$ & $109.91(17)$ \\
\hline$C(221)$ & $-P(2)$ & $-C(9)$ & $108.28(18)$ & $C(1)$ & $-P(2)$ & $-C(211)$ & $113.51(18)$ \\
\hline
\end{tabular}




\begin{tabular}{|c|c|c|c|c|c|c|c|}
\hline$C(221)$ & $-P(2)$ & $-C(211)$ & $103.94(18)$ & $C(9)$ & $-P(2)$ & $-c(211)$ & $108.73(17)$ \\
\hline$P(2)$ & $-C(1)$ & $-S(1)$ & $111.62(19)$ & $P(2)$ & $-C(1)$ & - Rh & $100.76(16)$ \\
\hline$S(1)$ & $-C(1)$ & $-\mathrm{Rh}$ & $111.41(16)$ & $P(2)$ & $-C(1)$ & $-H(1)$ & $111(3)$ \\
\hline$S(1)$ & $-C(1)$ & $-H(1)$ & $106(3)$ & $\mathrm{Rh}$ & $-C(1)$ & $-H(1)$ & $116(3)$ \\
\hline$C(3)$ & $-c(2)$ & $-C(7)$ & $121.1(4)$ & $C(3)$ & $-C(2)$ & $-S(1)$ & $119.0(3)$ \\
\hline$C(7)$ & $-C(2)$ & $-S(1)$ & $119.7(3)$ & $C(2)$ & $-C(3)$ & $-C(4)$ & $118.6(5)$ \\
\hline$C(5)$ & $-C(4)$ & $-c(3)$ & $121.2(5)$ & $C(6)$ & $-C(5)$ & $-C(4)$ & $119.1(5)$ \\
\hline$C(6)$ & $-c(5)$ & $-c(8)$ & $119.3(5)$ & $C(4)$ & $-c(5)$ & $-c(8)$ & $121.7(5)$ \\
\hline$C(5)$ & $-C(6)$ & $-C(7)$ & $120.8(5)$ & $C(6)$ & $-C(7)$ & $-c(2)$ & $119.2(4)$ \\
\hline$C(10)$ & $-C(9)$ & $-C(14)$ & $119.1(3)$ & $C(10)$ & $-C(9)$ & $-P(2)$ & $117.0(3)$ \\
\hline$C(14)$ & $-C(9)$ & $-P(2)$ & $123.8(3)$ & $C(11)$ & $-C(10)$ & $-C(9)$ & $120.9(4)$ \\
\hline$C(12)$ & $-C(11)$ & $-c(10)$ & $120.1(4)$ & $C(11)$ & $-C(12)$ & $-C(13)$ & $119.8(4)$ \\
\hline$C(12)$ & $-C(13)$ & $-C(14)$ & $122.0(4)$ & $c(13)$ & $-C(14)$ & $-C(9)$ & $118.0(3)$ \\
\hline$C(13)$ & $-C(14)$ & $-P(1)$ & $119.1(3)$ & $C(9)$ & $-C(14)$ & $-P(1)$ & $122.9(3)$ \\
\hline$C(16)$ & $-C(15)$ & $-C(22)$ & $124.5(4)$ & $C(16)$ & $-C(15)$ & - $R h$ & $70.44(19)$ \\
\hline$C(22)$ & $-C(15)$ & $-\mathrm{Rh}$ & $112.0(2)$ & $C(15)$ & $-C(16)$ & $-C(17)$ & $124.6(4)$ \\
\hline$C(15)$ & $-c(16)$ & $-\mathrm{Rh}$ & $72.5(2)$ & $C(17)$ & $-C(16)$ & - Rh & $110.4(2)$ \\
\hline$C(16)$ & $-C(17)$ & $-C(18)$ & $115.4(3)$ & $C(19)$ & $-C(18)$ & $-C(17)$ & $113.4(3)$ \\
\hline$C(20)$ & $-C(19)$ & $-C(18)$ & $124.2(4)$ & $C(20)$ & $-C(19)$ & - Rh & $70.4(2)$ \\
\hline$C(18)$ & $-C(19)$ & $-\mathrm{Rh}$ & $110.9(2)$ & $C(19)$ & $-C(20)$ & $-C(21)$ & $128.0(4)$ \\
\hline$C(19)$ & $-C(20)$ & $-\mathrm{Rh}$ & $74.5(2)$ & $C(21)$ & $-C(20)$ & $-\mathrm{Rh}$ & $106.0(2)$ \\
\hline$C(20)$ & $-C(21)$ & $-c(22)$ & $114.0(3)$ & $C(15)$ & $-C(22)$ & $-C(21)$ & $112.7(3)$ \\
\hline$C(112)$ & $-C(111)$ & $-C(116)$ & $117.8(3)$ & $C(112)$ & $-C(111)$ & $-P(1)$ & $118.7(3)$ \\
\hline$C(116)$ & $-C(111)$ & $-P(1)$ & $123.3(3)$ & $C(113)$ & $-C(112)$ & $-C(111)$ & $121.0(4)$ \\
\hline$C(114)$ & $-C(113)$ & $-C(112)$ & $119.8(4)$ & $C(115)$ & $-C(114)$ & $-C(113)$ & $120.3(4)$ \\
\hline$C(114)$ & $-C(115)$ & $-C(116)$ & $120.5(4)$ & $C(115)$ & $-C(116)$ & $-C(111)$ & $120.6(4)$ \\
\hline$C(126)$ & $-C(121)$ & $-C(122)$ & $118.2(4)$ & $C(126)$ & $-C(121)$ & $-P(1)$ & $123.2(3)$ \\
\hline$C(122)$ & $-C(121)$ & $-P(1)$ & $118.5(3)$ & $C(123)$ & $-C(122)$ & $-\mathrm{C}(121)$ & $120.5(4)$ \\
\hline$C(124)$ & $-c(123)$ & $-C(122)$ & $120.6(4)$ & $\mathrm{C}(123)$ & $-C(124)$ & $-C(125)$ & $119.6(4)$ \\
\hline$C(124)$ & $-C(125)$ & $-C(126)$ & $120.4(5)$ & $C(121)$ & $-C(126)$ & $-C(125)$ & $120.6(4)$ \\
\hline$C(216)$ & $-C(211)$ & $-C(212)$ & $118.9(4)$ & $C(216)$ & $-C(211)$ & $-P(2)$ & $121.2(4)$ \\
\hline$C(212)$ & $-C(211)$ & $-P(2)$ & $119.9(3)$ & $C(213)$ & $-C(212)$ & $-C(211)$ & $120.1(5)$ \\
\hline$C(214)$ & $-C(213)$ & $-c(212)$ & $120.4(6)$ & $C(213)$ & $-C(214)$ & $-C(215)$ & $120.5(5)$ \\
\hline$C(214)$ & $-C(215)$ & $-C(216)$ & $120.4(5)$ & $C(211)$ & $-C(216)$ & $-C(215)$ & $119.7(5)$ \\
\hline$C(222)$ & $-C(221)$ & $-C(226)$ & $119.7(4)$ & $C(222)$ & $-C(221)$ & $-P(2)$ & $121.8(3)$ \\
\hline$C(226)$ & $-C(221)$ & $-P(2)$ & $118.3(3)$ & $C(221)$ & $-C(222)$ & $-C(223)$ & $120.3(4)$ \\
\hline$C(224)$ & $-C(223)$ & $-C(222)$ & $119.4(4)$ & $C(223)$ & $-C(224)$ & $-C(225)$ & $120.8(4)$ \\
\hline$C(226)$ & $-C(225)$ & $-C(224)$ & $120.0(4)$ & $C(225)$ & $-C(226)$ & $-C(221)$ & $119.7(4)$ \\
\hline
\end{tabular}


(C) 2003 American Chemical Society, Organometallics, Zurawinski om030343g Supporting Info Page 7

*PF6

\begin{tabular}{|c|c|c|c|}
\hline (3) & $-P(3)$ & $-F(2)$ & $90.5(3)$ \\
\hline$F(2)$ & $-P(3)$ & $-F(5)$ & $173.9(3)$ \\
\hline$F(2)$ & $-P(3)$ & $-F(1)$ & $91.8(3)$ \\
\hline$E(3)$ & $-P(3)$ & $-F(6)$ & $179.0(3)$ \\
\hline$F(5)$ & $-P(3)$ & $-F(6)$ & $87.6(3)$ \\
\hline$F(3)$ & $-P(3)$ & $-F(4)$ & $90.8(2)$ \\
\hline$F(5)$ & $-P(3)$ & $-F(4)$ & $88.7(3)$ \\
\hline (6) & $-P(3)$ & $-F(4)$ & $89.2(2)$ \\
\hline
\end{tabular}

$\begin{array}{ll}F(3)-P(3)-F(5) & 93.4(3) \\ F(3)-P(3)-F(1) & 91.5(2) \\ F(5)-P(3)-F(1) & 92.8(3) \\ F(2)-P(3)-F(6) & 88.5(2) \\ F(1)-P(3)-F(6) & 88.56(19) \\ F(2)-P(3)-F(4) & 86.5(2) \\ E(1)-P(3)-F(4) & 177.2(2)\end{array}$

Table S4. Anisotropic displacement parameters ( $\$ 2 x 103$, the anisotropic displacement factor exponent takes the form:

$\exp \left[-2 p 2\left(U 11 h 2 a^{*} 2+U 22 k 2 b^{*} 2+U 3312 c^{*} 2+2 U 12 h k a^{*} b^{*}+2 U 13 h l a * c^{*}+\right.\right.$ $\left.\left.2 U 23 \mathrm{klb}^{*} \mathrm{c}^{*}\right)\right]$

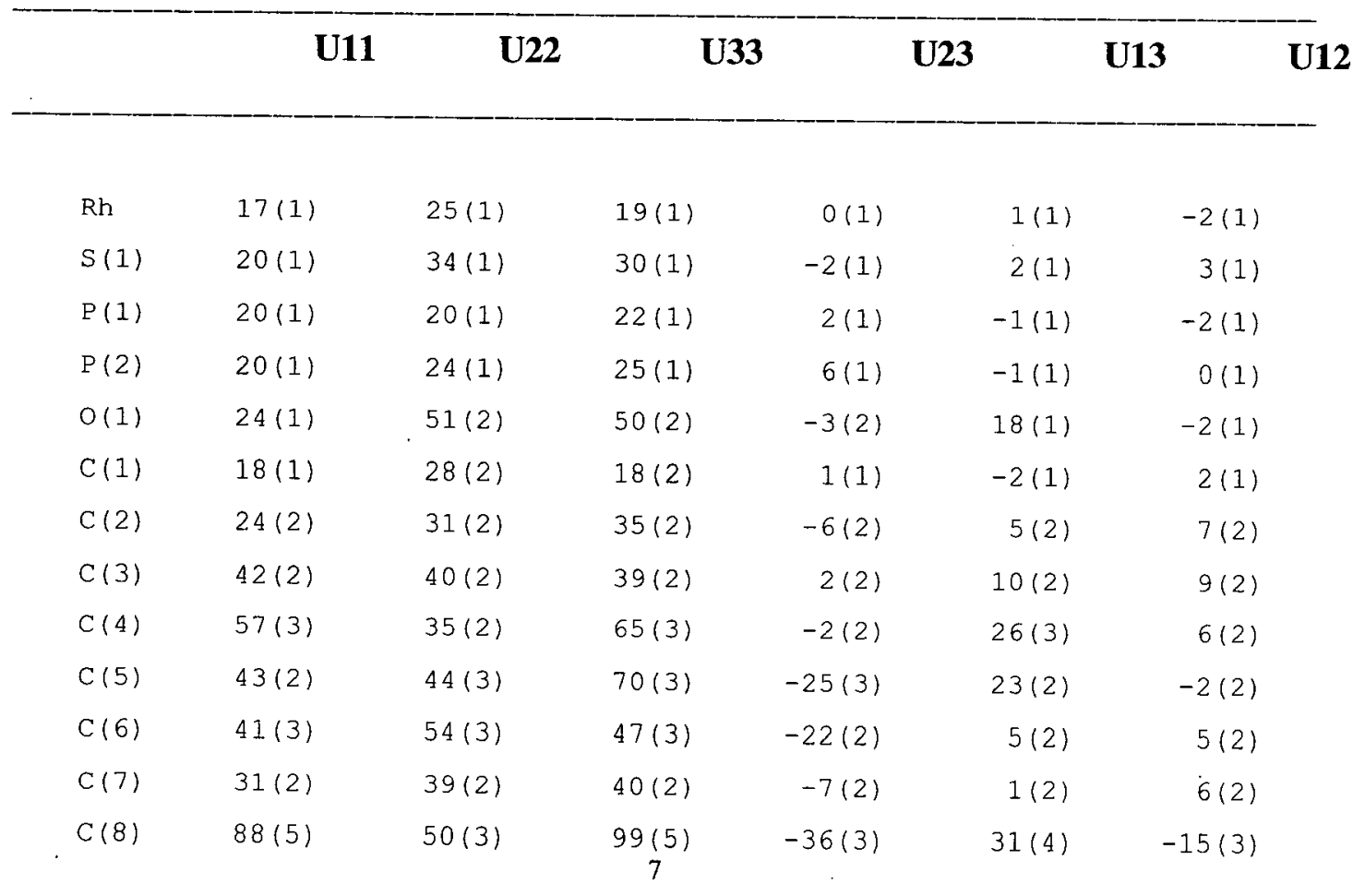


(C) 2003 American Chemical Society, Organometallics, Zurawinski om030343g Supporting Info Page 8

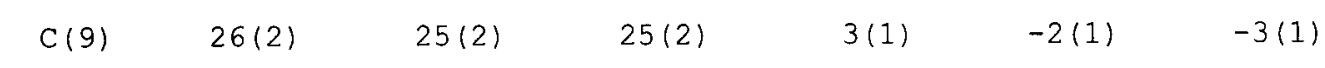

$\begin{array}{llllll}\text { U11 } & \text { U22 } & \text { U33 } & \text { U23 } & \text { U13 } & \text { U12 }\end{array}$

\begin{tabular}{|c|c|c|c|c|c|c|}
\hline$C(10)$ & $40(2)$ & $34(2)$ & $33(2)$ & $13(2)$ & $-8(2)$ & $-16(2)$ \\
\hline$C(11)$ & $45(3)$ & $43(3)$ & $46(3)$ & $9(2)$ & $-10(2)$ & $-22(2)$ \\
\hline$C(12)$ & $38(2)$ & $50(3)$ & $34(2)$ & $1(2)$ & $-5(2)$ & $-21(2)$ \\
\hline$C(13)$ & $32(2)$ & $35(2)$ & $24(2)$ & $5(2)$ & $-5(2)$ & $-11(2)$ \\
\hline$C(14)$ & $19(2)$ & $22(2)$ & $26(2)$ & $4(1)$ & $0(1)$ & $-2(1)$ \\
\hline$C(15)$ & $21(2)$ & $37(2)$ & $19(2)$ & $2(1)$ & $2(1)$ & $-5(2)$ \\
\hline$C(16)$ & $22(2)$ & $39(2)$ & $22(2)$ & $0(2)$ & $9(1)$ & $-3(2)$ \\
\hline$C(17)$ & $21(2)$ & $59(3)$ & $30(2)$ & $2(2)$ & $6(2)$ & $6(2)$ \\
\hline$C(18)$ & $19(2)$ & $62(3)$ & $29(2)$ & $1(2)$ & $-4(1)$ & $-1(2)$ \\
\hline$C(19)$ & $17(2)$ & $38(2)$ & $28(2)$ & $-2(2)$ & $-5(1)$ & $-4(2)$ \\
\hline$C(20)$ & $27(2)$ & $38(2)$ & $22(2)$ & $-2(2)$ & $1(1)$ & $-9(2)$ \\
\hline$C(21)$ & $46(2)$ & $32(2)$ & $35(2)$ & $o(2)$ & $-8(2)$ & $-13(2)$ \\
\hline$C(22)$ & $42(2)$ & $38(2)$ & $27(2)$ & $5(2)$ & $2(2)$ & $-17(2)$ \\
\hline$C(111)$ & $25(2)$ & $22(2)$ & $19(2)$ & $1(1)$ & $-2(1)$ & $-4(1)$ \\
\hline$C(112)$ & $39(2)$ & $27(2)$ & $30(2)$ & $3(2)$ & $-1(2)$ & $3(2)$ \\
\hline$C(113)$ & $44(2)$ & $30(2)$ & $37(2)$ & $-5(2)$ & $2(2)$ & $5(2)$ \\
\hline$C(114)$ & $43(2)$ & $44(2)$ & $26(2)$ & $-4(2)$ & $6(2)$ & $-7(2)$ \\
\hline$C(115)$ & $46(2)$ & $38(2)$ & $26(2)$ & $7(2)$ & $-4(2)$ & $-2(2)$ \\
\hline$C(116)$ & $36(2)$ & $26(2)$ & $29(2)$ & $2(2)$ & $-3(2)$ & $5(2)$ \\
\hline$C(121)$ & $25(2)$ & $27(2)$ & $23(2)$ & $-2(1)$ & $-3(1)$ & $6(1)$ \\
\hline$C(122)$ & $33(2)$ & $30(2)$ & $42(2)$ & $-3(2)$ & $-2(2)$ & $1(2)$ \\
\hline$C(123)$ & $45(3)$ & $24(2)$ & $58(3)$ & $2(2)$ & $-7(2)$ & $1(2)$ \\
\hline$C(124)$ & $43(3)$ & $33(2)$ & $70(3)$ & $2(2)$ & $-13(2)$ & $15(2)$ \\
\hline$C(125)$ & $29(2)$ & $53(3)$ & $77(4)$ & $3(3)$ & $-5(2)$ & $13(2)$ \\
\hline$C(126)$ & $27(2)$ & $39(2)$ & $53(3)$ & $5(2)$ & $-2(2)$ & $2(2)$ \\
\hline$C(211)$ & $27(2)$ & $38(2)$ & $32(2)$ & $16(2)$ & $-7(2)$ & $-9(2)$ \\
\hline$C(212)$ & $54(3)$ & $49(3)$ & $34(2)$ & $9(2)$ & $9(2)$ & $-10(2)$ \\
\hline$C(213)$ & $78(4)$ & $73(4)$ & $37(3)$ & $19(3)$ & $9(3)$ & $-10(3)$ \\
\hline$C(214)$ & $66(4)$ & $71(4)$ & $48(3)$ & $32(3)$ & $2(3)$ & $-8(3)$ \\
\hline$C(215)$ & $67(4)$ & $45(3)$ & $82(4)$ & $38(3)$ & $-3(3)$ & $3(3)$ \\
\hline$C(216)$ & $54(3)$ & $45(3)$ & $53(3)$ & $21(2)$ & $6(2)$ & $11(2)$ \\
\hline$C(221)$ & $29(2)$ & $26(2)$ & $30(2)$ & $5(2)$ & $2(2)$ & $5(2)$ \\
\hline$C(222)$ & $36(2)$ & $32(2)$ & $40(2)$ & $0(2)$ & $0(2)$ & $8(2)$ \\
\hline$C(223)$ & $55(3)$ & $35(2)$ & $42(3)$ & $2(2)$ & $8(2)$ & $18(2)$ \\
\hline$C(224)$ & $47(3)$ & $33(2)$ & $64(3)$ & $14(2)$ & $17(2)$ & $21(2)$ \\
\hline
\end{tabular}


(C) 2003 American Chemical Society, Organometallics, Zurawinski om030343g Supporting Info Page 9

$\begin{array}{rrrrrrr}C(225) & 32(2) & 37(2) & 66(3) & 3(2) & -3(2) & 11(2) \\ C(226) & 30(2) & 33(2) & 47(2) & 4(2) & 2(2) & 6(2)\end{array}$

\section{$\begin{array}{llllll}\text { U11 } & \text { U22 } & \text { U33 } & \text { U23 } & \text { U13 } & \text { U12 }\end{array}$}

$\begin{array}{rrrrrrr}P(3) & 33(1) & 49(1) & 46(1) & -10(1) & 8(1) & -8(1) \\ F(1) & 74(2) & 140(4) & 78(2) & 61(2) & -21(2) & -49(2) \\ F(2) & 73(2) & 97(3) & 87(3) & -25(2) & 16(2) & 32(2) \\ F(3) & 67(2) & 220(5) & 50(2) & 3(3) & -12(2) & -80(3) \\ F(4) & 82(3) & 63(2) & 117(3) & 27(2) & -7(3) & -29(2) \\ F(5) & 102(4) & 123(4) & 155(5) & -84(4) & 49(3) & -13(3) \\ F(6) & 47(2) & 65(2) & 100(3) & 8(2) & -23(2) & -11(2)\end{array}$

Table S5. Hydrogen coordinates (x104) and isotropic displacement parameters (Å2x103)

\begin{tabular}{|c|c|c|c|c|}
\hline & $\mathbf{x}$ & $\mathbf{y}$ & $\mathbf{Z}$ & $\mathbf{U}(\mathbf{e q})$ \\
\hline $\mathrm{H}(1)$ & $7380(30)$ & $8260(30)$ & $2669(11)$ & $32(12)$ \\
\hline $\mathrm{H}(3)$ & 6196 & 10643 & 2295 & 49 \\
\hline $\mathrm{H}(4)$ & 6688 & 11993 & 2558 & 63 \\
\hline $\mathrm{H}(6)$ & 7315 & 10583 & 3399 & 57 \\
\hline $\mathrm{H}(7)$ & 6837 & 9228 & 3143 & 44 \\
\hline$H(8 A)$ & 7786 & 12626 & 3031 & 119 \\
\hline $\mathrm{H}(8 \mathrm{~B})$ & 7680 & 12132 & 3390 & 119 \\
\hline $\mathrm{H}(8 \mathrm{C})$ & 6830 & 12675 & 3230 & 119 \\
\hline $\mathrm{H}(10)$ & 5378 & 5843 & 2404 & 43 \\
\hline $\mathrm{H}(11)$ & 4143 & 5550 & 2045 & 54 \\
\hline $\mathrm{H}(12)$ & 4001 & 6345 & 1542 & 49 \\
\hline $\mathrm{H}(13)$ & 5075 & 7455 & 1398 & 36 \\
\hline $\mathrm{H}(15)$ & 8003 & 9291 & 1514 & 31 \\
\hline$H(16)$ & 8580 & 7853 & 1575 & 33 \\
\hline$H(17 A)$ & 10334 & $\begin{array}{l}8291 \\
9\end{array}$ & 1663 & 44 \\
\hline
\end{tabular}


(C) 2003 American Chemical Society, Organometallics, Zurawinski om030343g Supporting Info Page 10

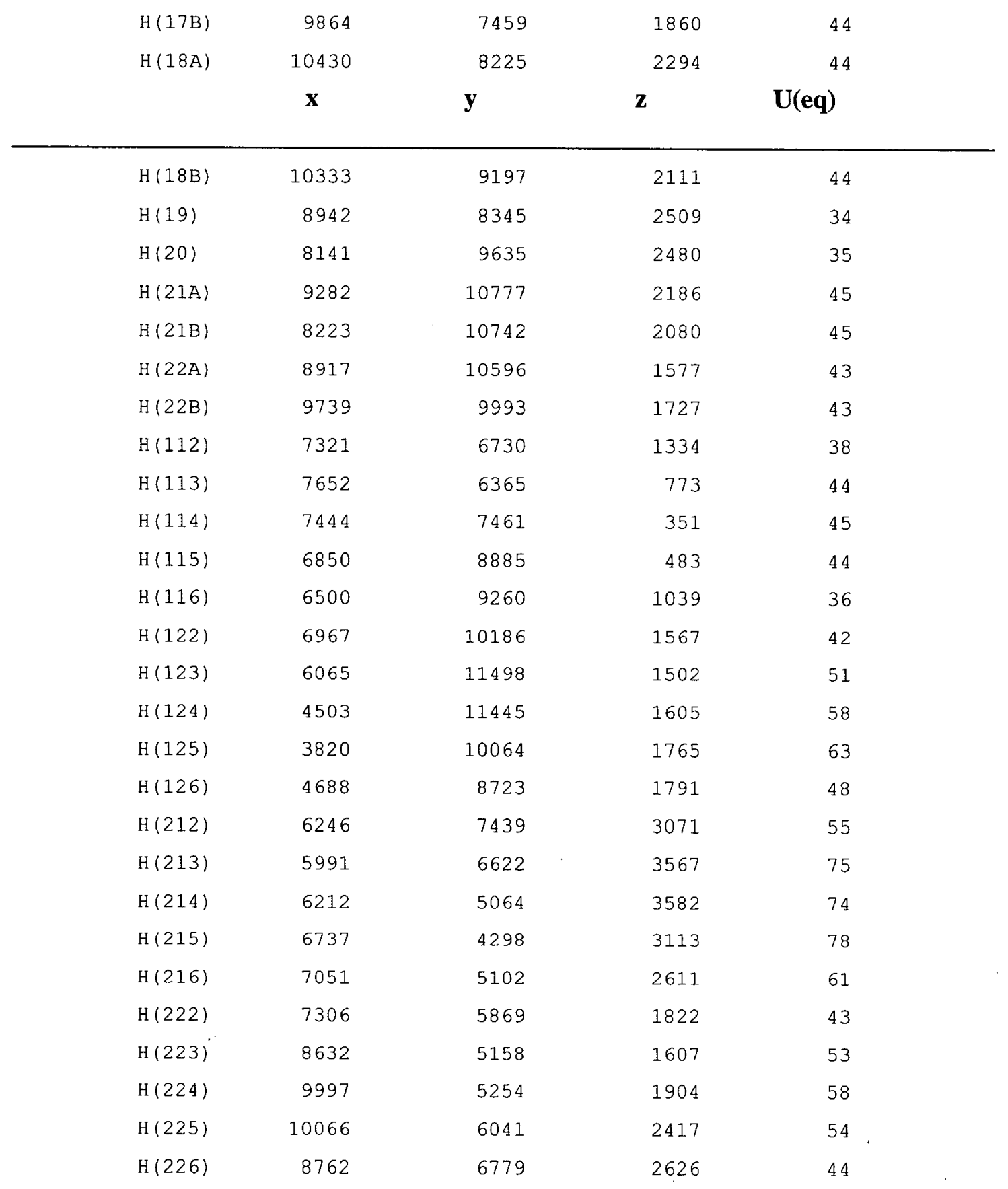




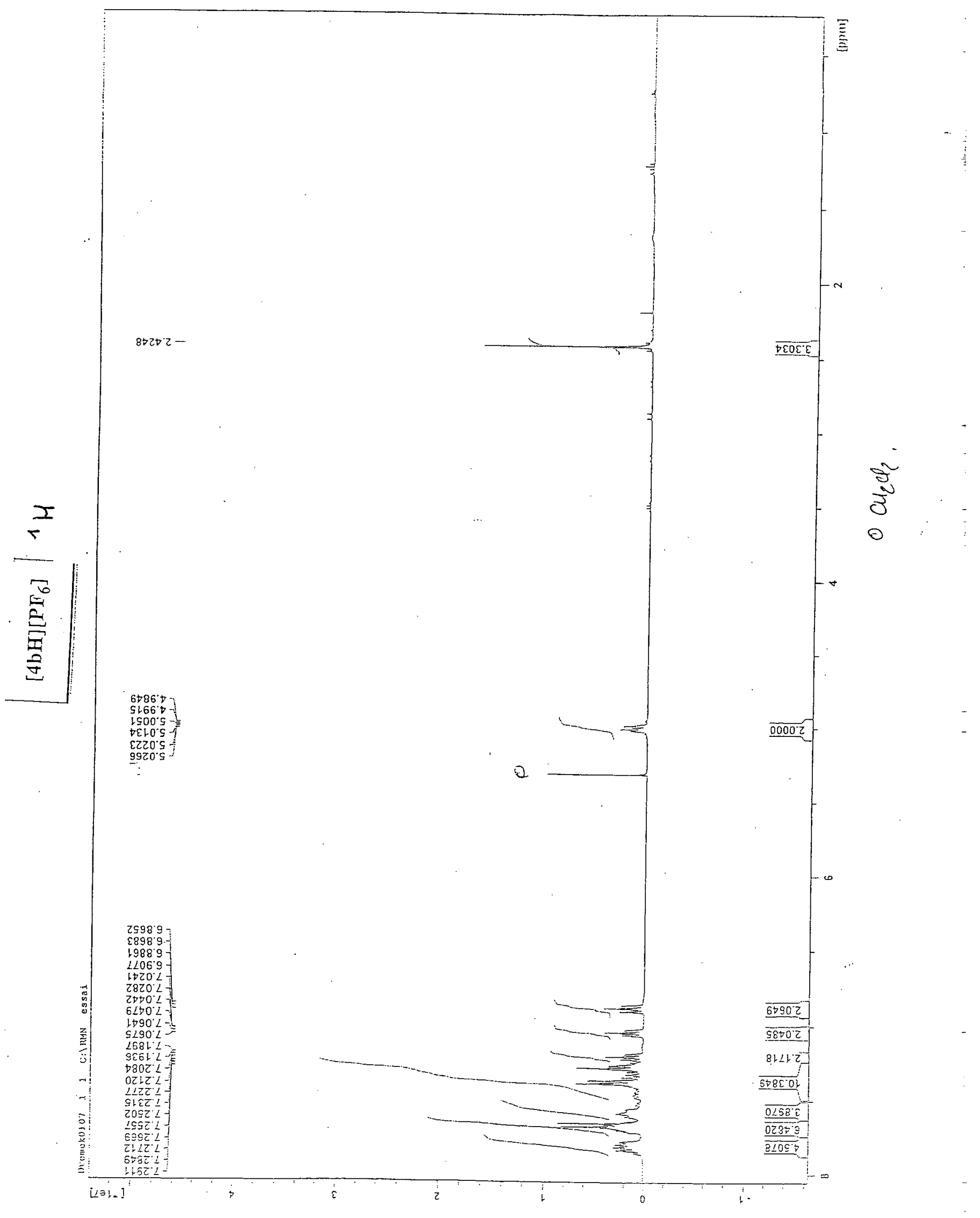


(C) 2003 American Chemical Society, Organometallics, Zurawinski om030343g Supporting Info Page 12
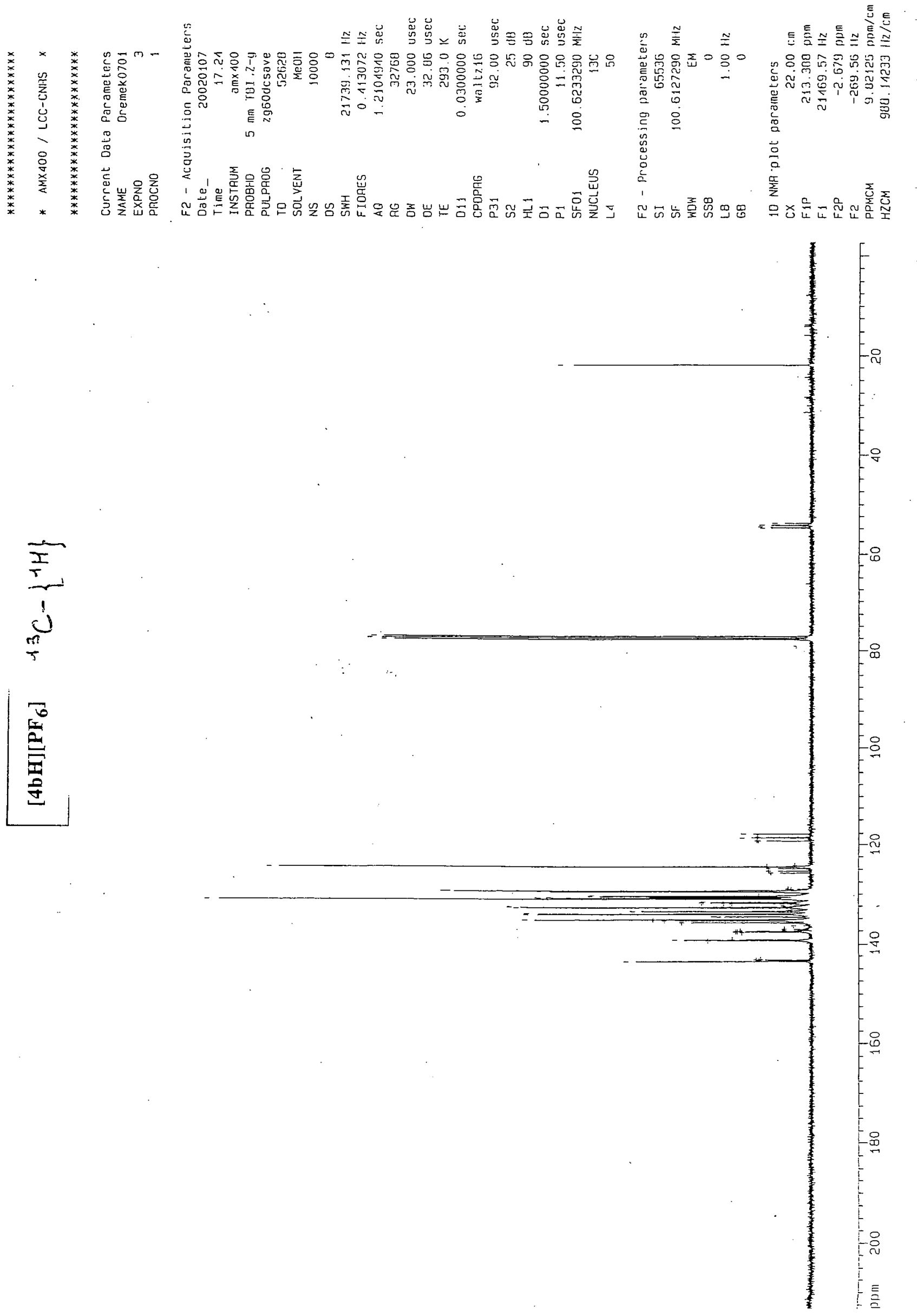
(C) 2003 American Chemical Society, Organometallics, Zurawinski om030343g Supporting Info Page 13
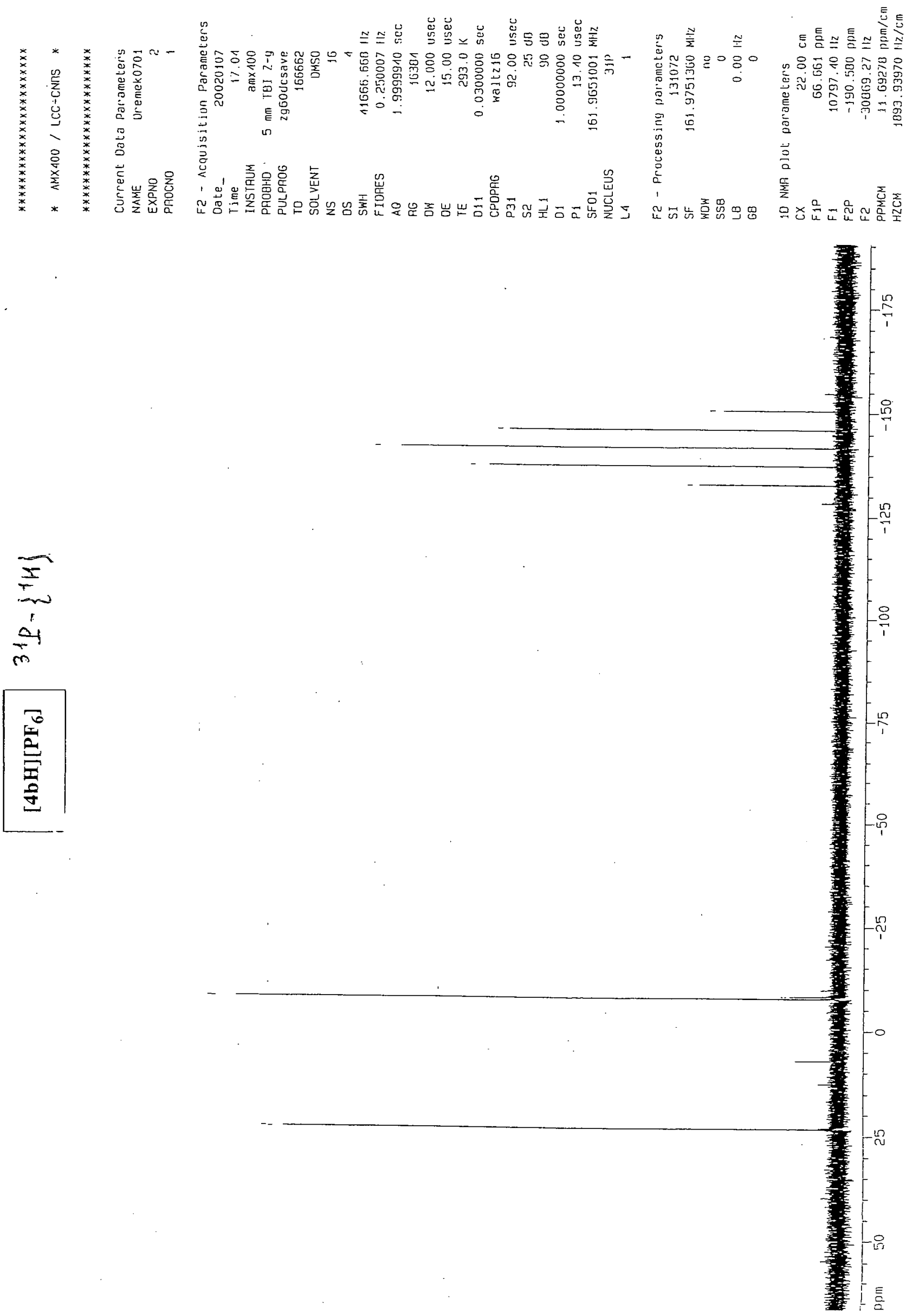
(C) 2003 American Chemical Society, Organometallics, Zurawinski om030343g Supporting Info Page 14
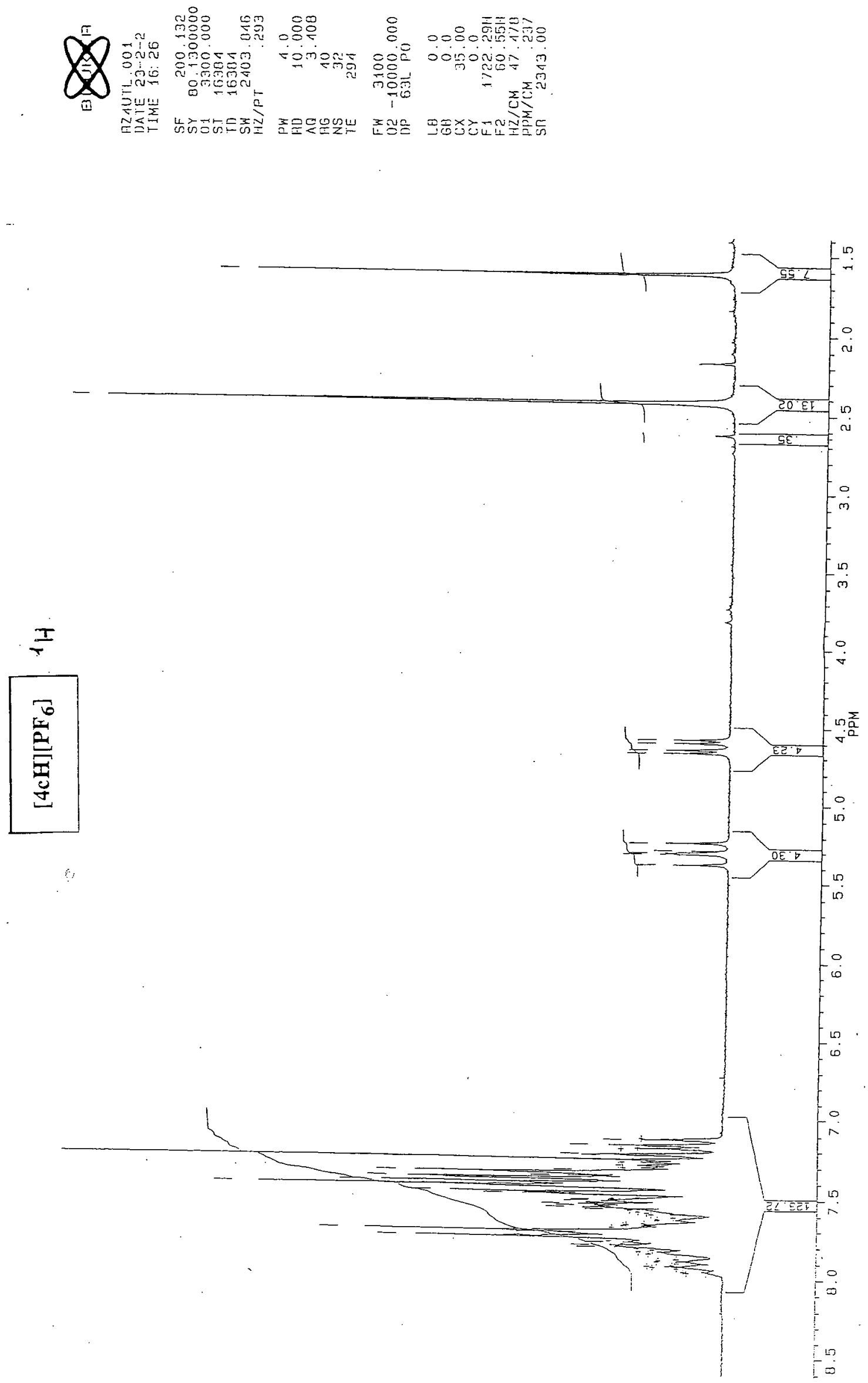
(C) 2003 American Chemical Society, Organometallics, Zurawinski om030343g Supporting Info Page 15

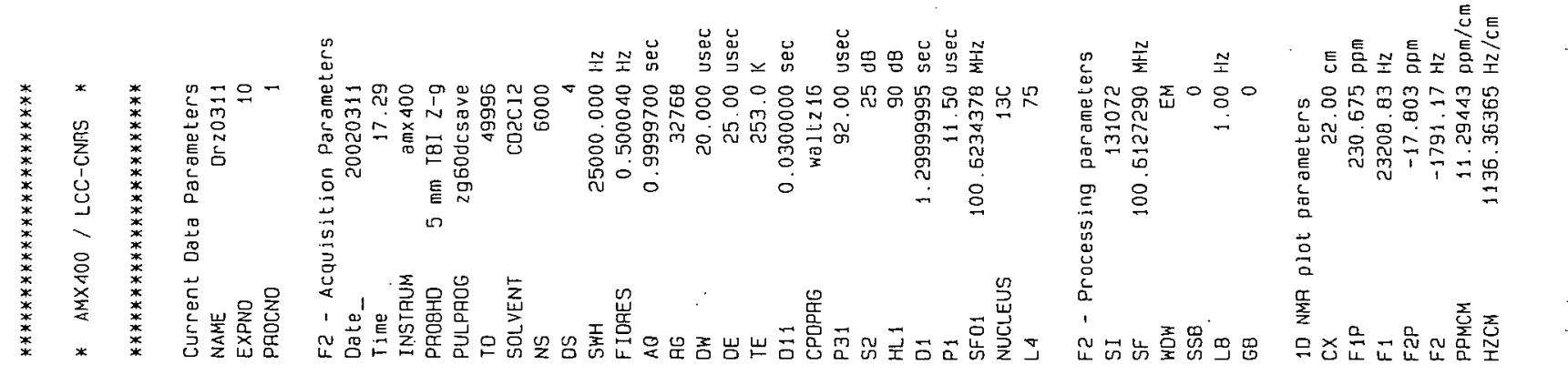
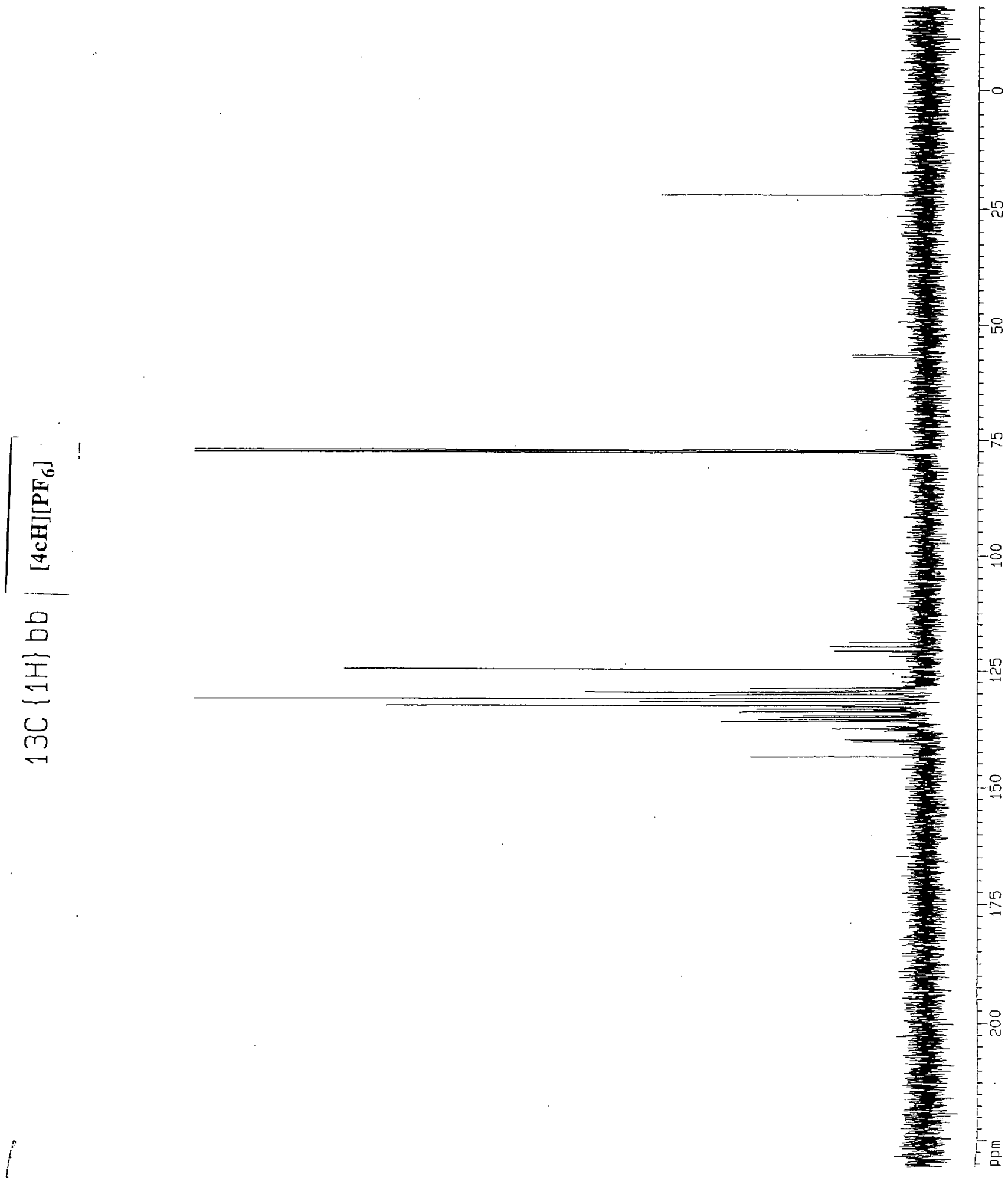
(C) 2003 American Chemical Society, Organometallics, Zurawinski om030343g Supporting Info Page 16
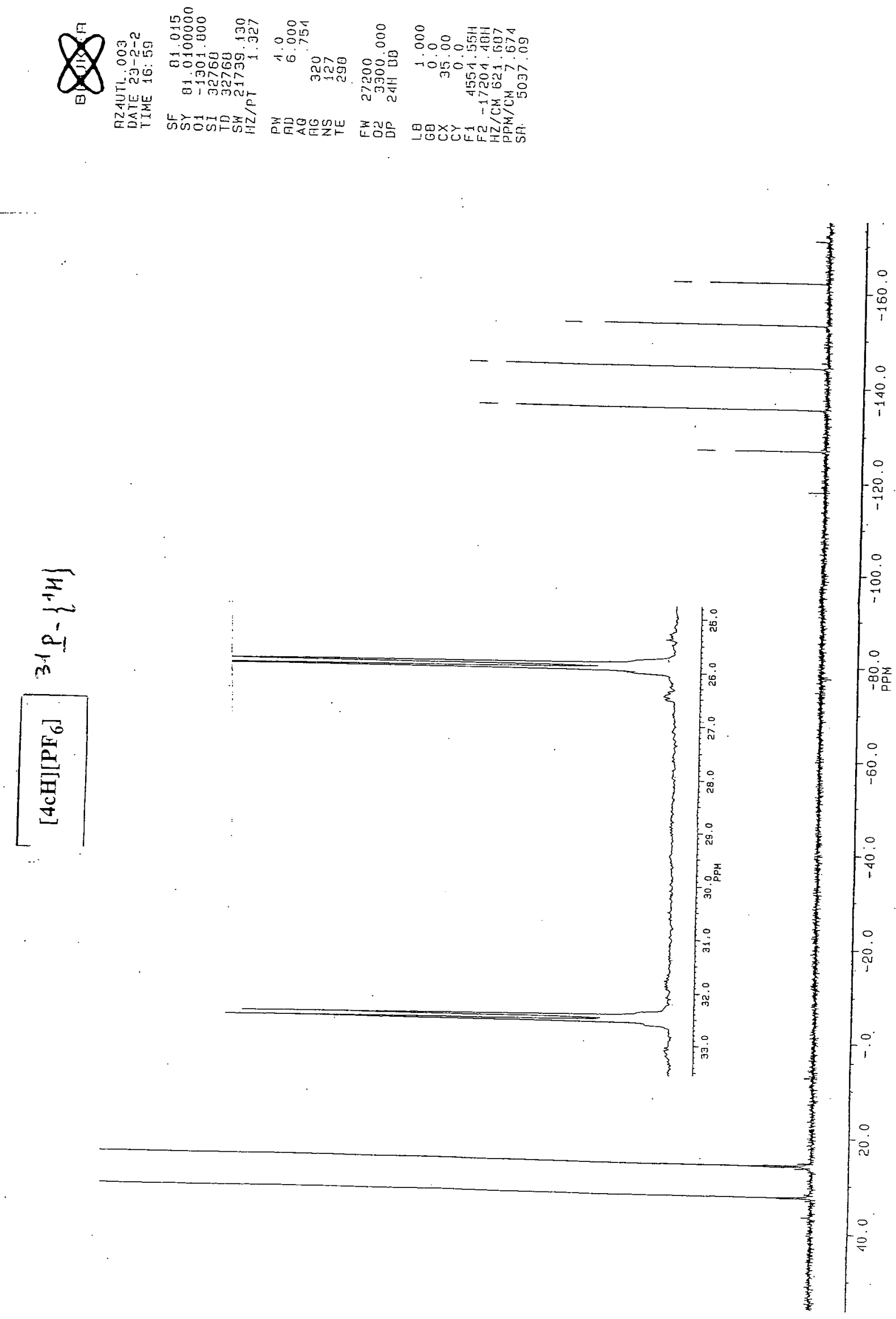
(C) 2003 American Chemical Society, Organometallics, Zurawinski om030343g Supporting Info Page 17
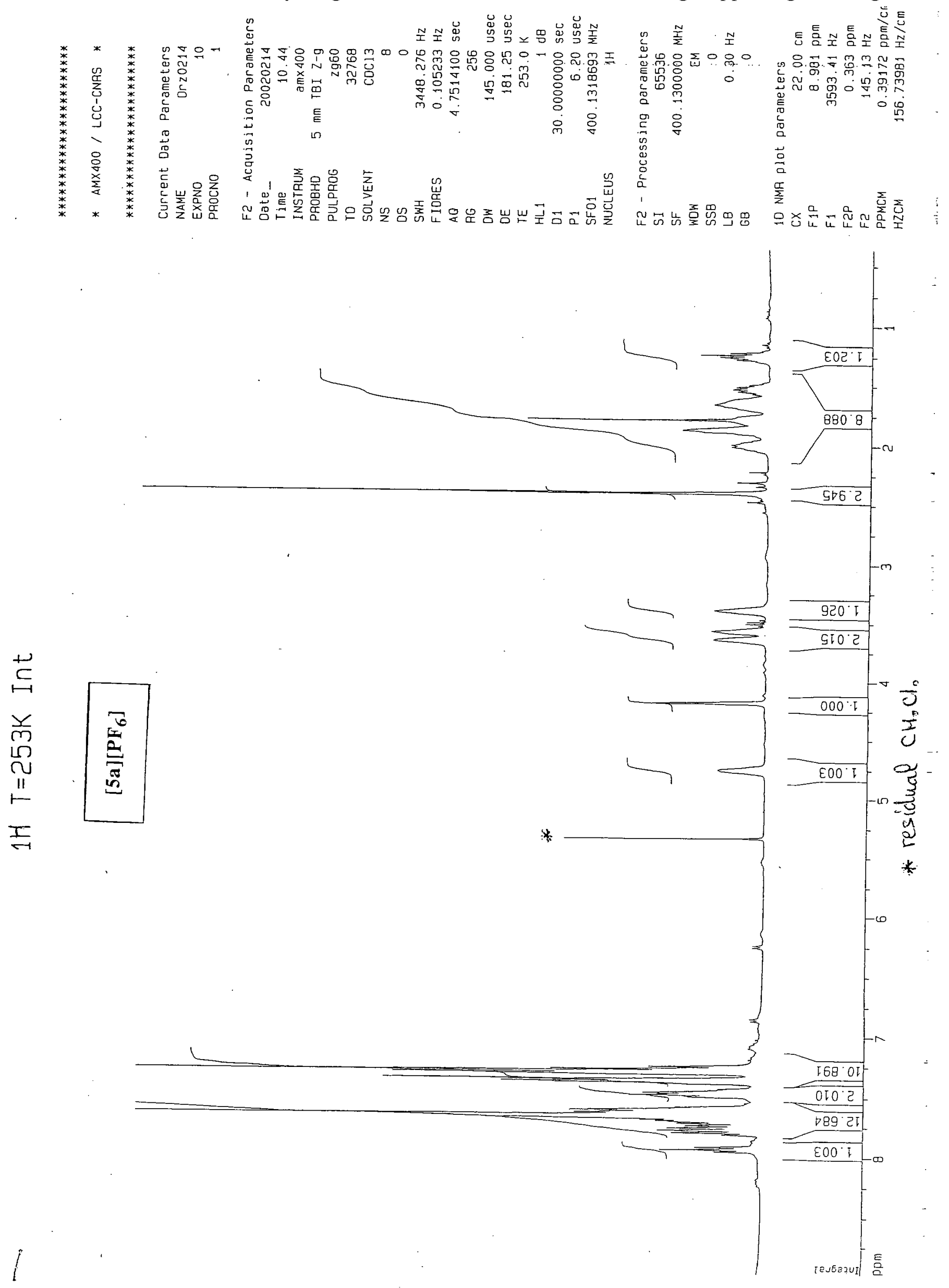
(C) 2003 American Chemical Society, Organometallics, Zurawinski om030343g Supporting Info Page 18
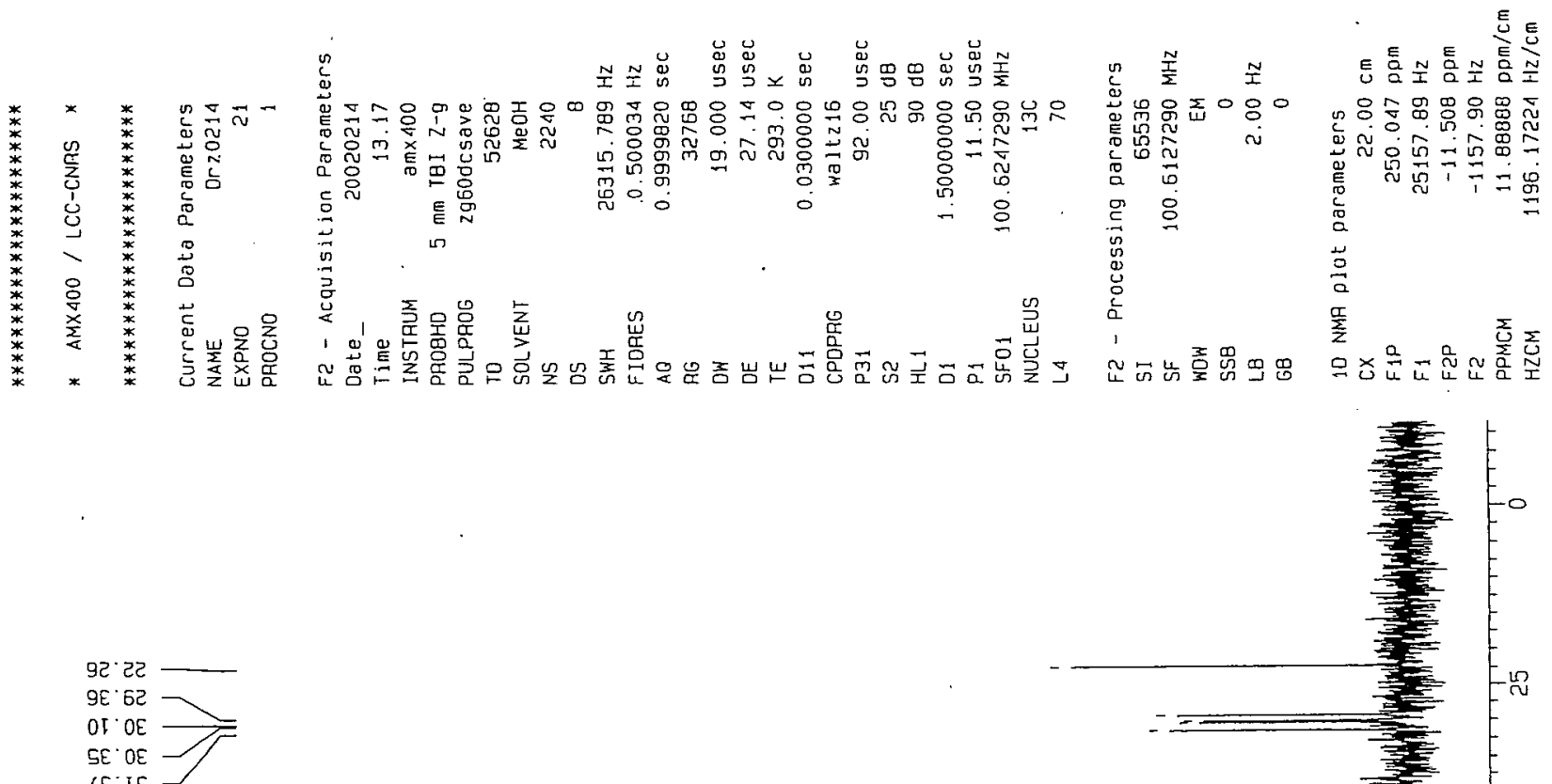

$\angle E^{\circ}$ IE
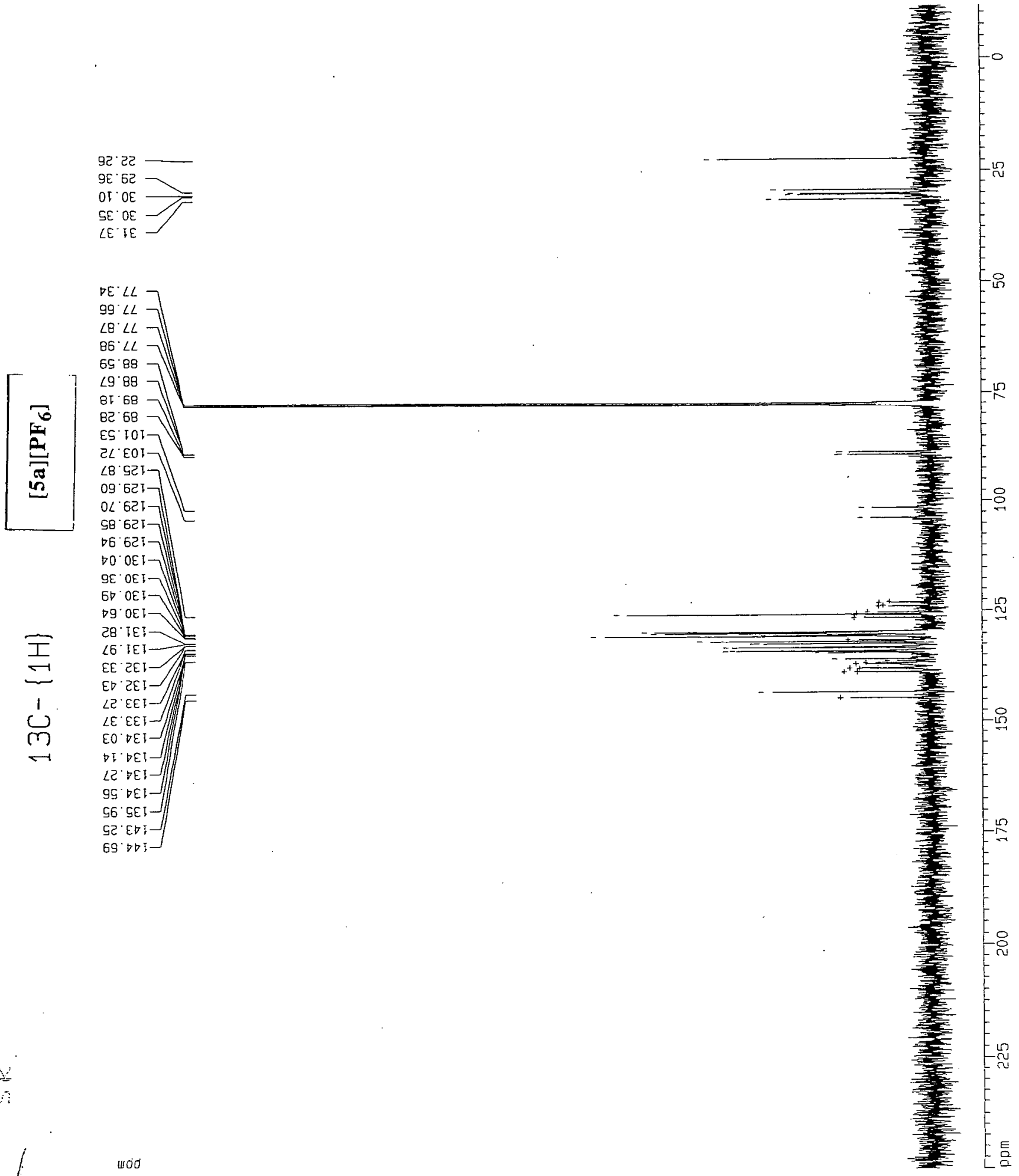
(C) 2003 American Chemical Society, Organometallics, Zurawinski om030343g Supporting Info Page 19

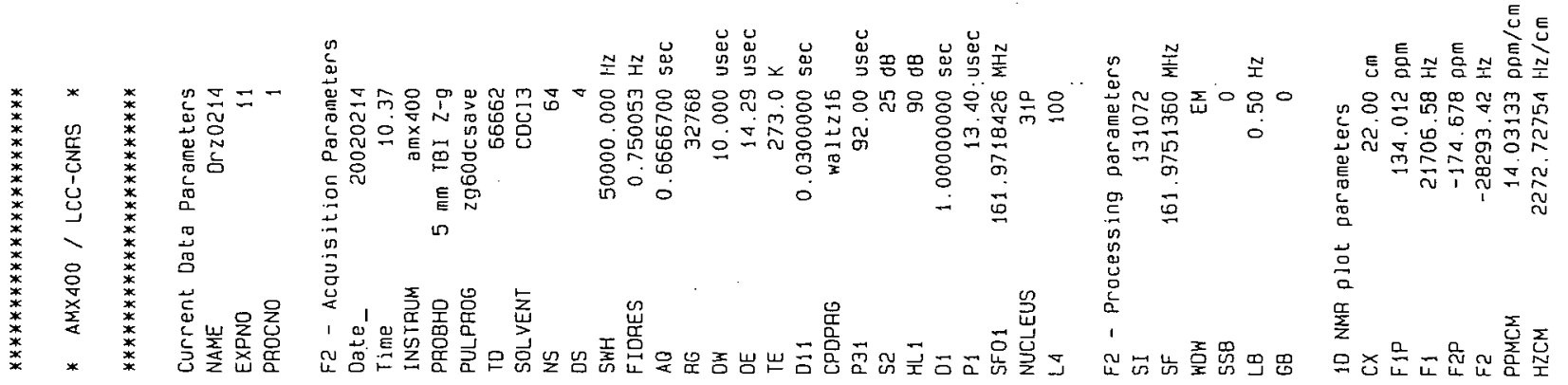
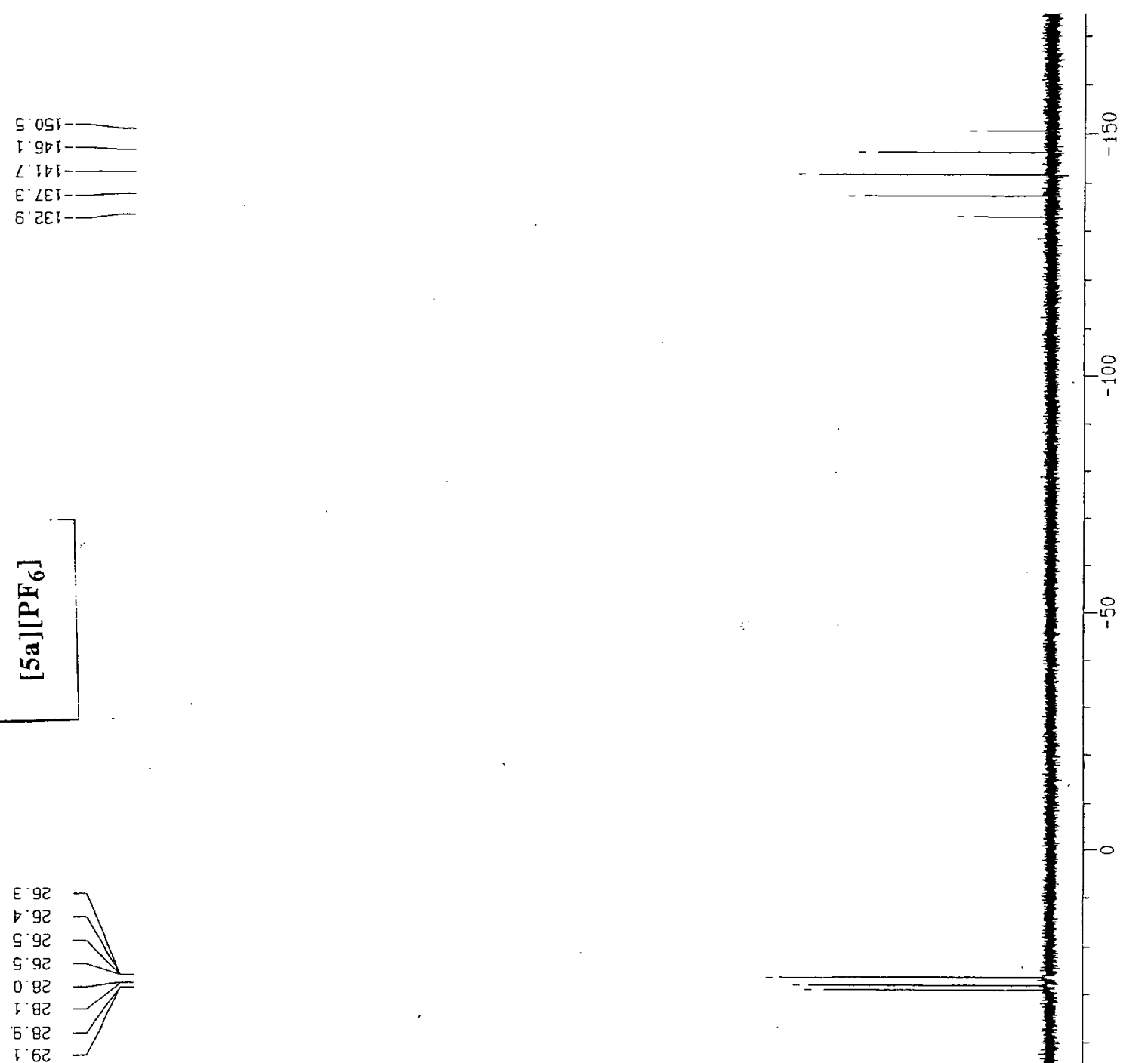
(C) 2003 American Chemical Society, Organometallics, Zurawinski om030343g Supporting Info Page 20

\begin{tabular}{l}
$\frac{1}{5}$ \\
\multirow{2}{*}{} \\
$\tilde{J}$ \\
$\tilde{\sigma}$ \\
$\bar{\sigma}$
\end{tabular}

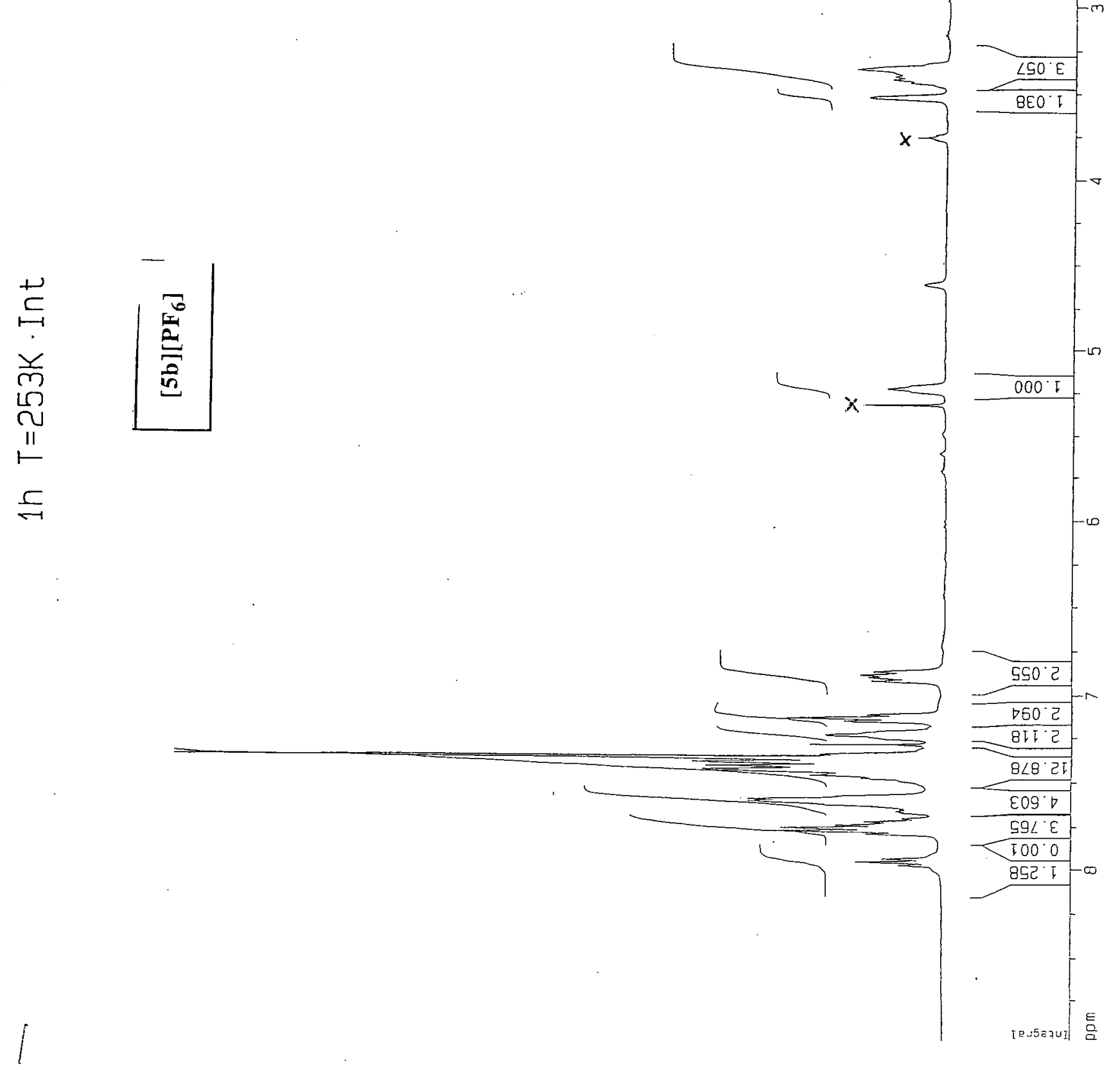




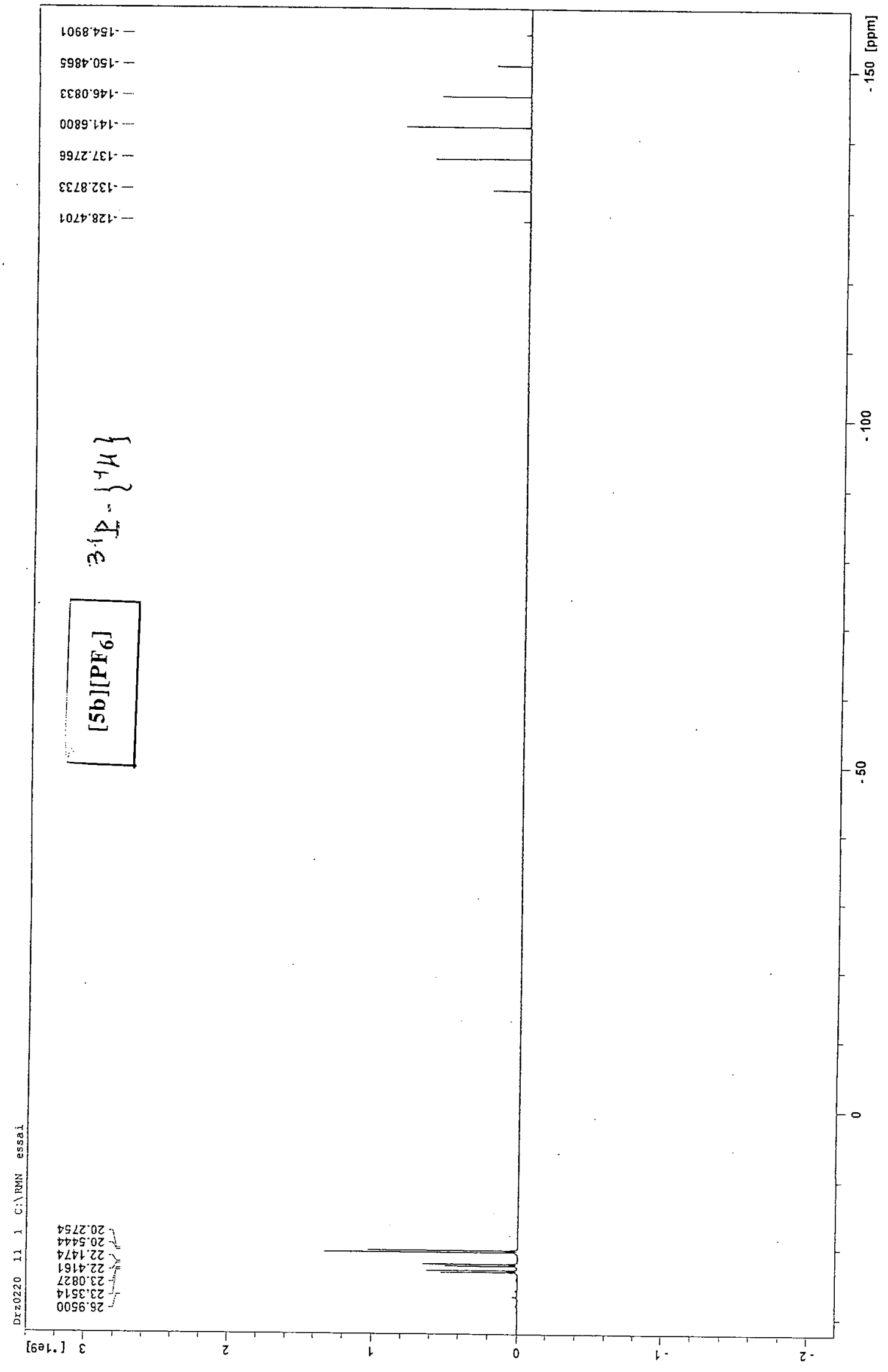


(C) 2003 American Chemical Society, Organometallics, Zurawinski om030343g Supporting Info Page 22

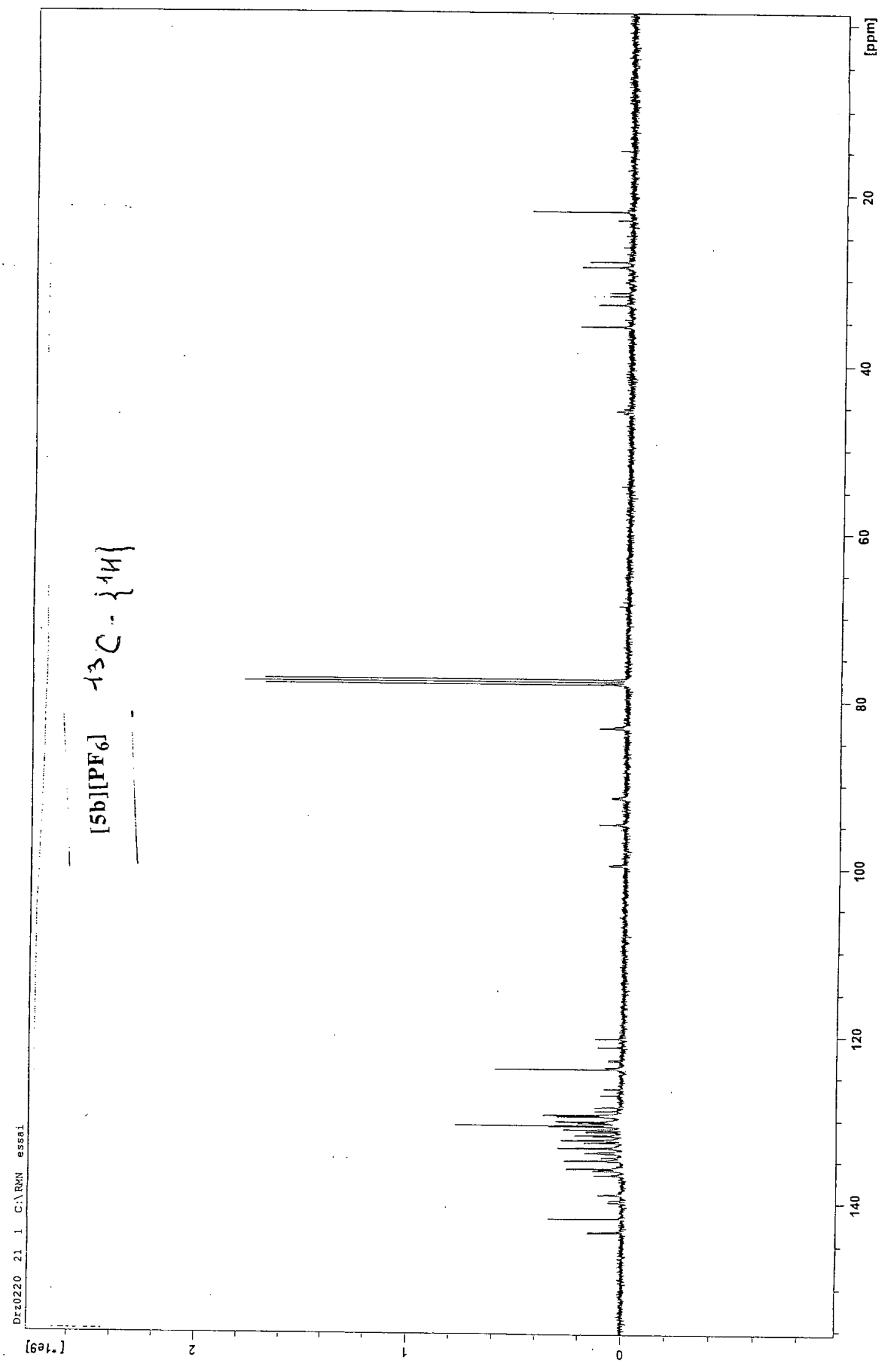

